Article

\title{
Effects of Achilles Tendon Moment Arm Length on Insertional Achilles Tendinopathy
}

\author{
Takuma Miyamoto ${ }^{1}$, Yasushi Shinohara ${ }^{2, *}$, Tomohiro Matsui ${ }^{3}$, Hiroaki Kurokawa ${ }^{1}$, \\ Akira Taniguchi ${ }^{1}$, Tsukasa Kumai ${ }^{4} \mathbb{D}$ and Yasuhito Tanaka ${ }^{1}$ (D) \\ 1 Orthopaedic Surgery, Nara Medical University, Nara 634-8521, Japan; k166136@naramed-u.ac.jp (T.M.); \\ blackandriver@hotmail.co.jp (H.K.); a-tani@naramed-u.ac.jp (A.T.); yatanaka@naramed-u.ac.jp (Y.T.) \\ 2 College of Sport and Health Science, Ritsumeikan University, Shiga 525-8577, Japan \\ 3 Orthopaedic Surgery, Saiseikai Nara Hospital, Nara 630-8145, Japan; t.matsui19810671@live.jp \\ 4 Faculty of Sport Sciences, Waseda University, Tokyo 169-8050, Japan; kumakumat@waseda.jp \\ * Correspondence: ysr15159@fc.ritsumei.ac.jp; Tel.: +81-77-561-2617
}

Received: 21 August 2020; Accepted: 22 September 2020; Published: 23 September 2020

check for updates

\begin{abstract}
Insertional Achilles tendinopathy (IAT) is caused by traction force of the tendon. The effectiveness of the suture bridge technique in correcting it is unknown. We examined the moment arm in patients with IAT before and after surgery using the suture bridge technique, in comparison to that of healthy individuals. We hypothesized that the suture bridge method influences the moment arm length. An IAT group comprising 10 feet belonging to 8 patients requiring surgical treatment for IAT were followed up postoperatively and compared with a control group comprising 15 feet of 15 healthy individuals with no ankle complaints or history of trauma or surgery. The ratio of the moment arm (MA) length/foot length was found to be statistically significant between the control group, the IAT group preoperatively and the IAT group postoperatively $(p<0.01)$. Despite no significant difference in the force between the control and preoperative IAT groups, a significantly higher force to the Achilles tendon was observed in the IAT group postoperatively compared to the other groups $(p<0.05)$. This study demonstrates that a long moment arm may be one of the causes of IAT, and the suture bridge technique may reduce the Achilles tendon moment arm.
\end{abstract}

Keywords: insertional Achilles tendinopathy; Achilles tendon moment arm; suture bridge method

\section{Introduction}

The Achilles tendon, attached to the posterior process of the calcaneus, is the largest and strongest tendon in the body. It is composed of the gastrocnemius muscle, originating from the condyle of the femur, and the soleus muscle, originating from the upper tibia. The gastrocnemius and soleus muscles, collectively known as the triceps surae, flex the ankle joint during gait. In the gait cycle, balance is maintained against gravity during the stance phase, and flexing the ankle joint during the toe-off phase provides a forward propulsive force [1].

The contraction of the triceps surae is exerted as a rotational force (torque) around the ankle axis, which can be calculated theoretically as the product of the muscle cross-section and the moment arm (MA) (Figure 1) [2-4].

In general, the length of the MA is known to change depending on the angles of plantarflexion and dorsiflexion of the ankle joint, which is different in each individual [2-4]. However, the length of the MA of the triceps surae has been reported to change depending on the angle of plantar- and dorsiflexion of the ankle joint [5-7]. Yet, it has also been reported that motion does not cause any change in the length of the MA [8,9], and that the plantarflexion moment length, from the plantarflexion region to the dorsiflexion region, is different in each individual, with little change caused by motion [2-4]. 
Thus, the changes in the length of the MA in motion has attracted great attention. Moreover, the shape of the foot has been shown to influence the Achilles tendon MA [10]. However, these studies examined the length of the MA only in healthy individuals and it has not been investigated in patients with Achilles tendon disorders.

\section{The contraction of the triceps surae $(\mathrm{Nm})$}

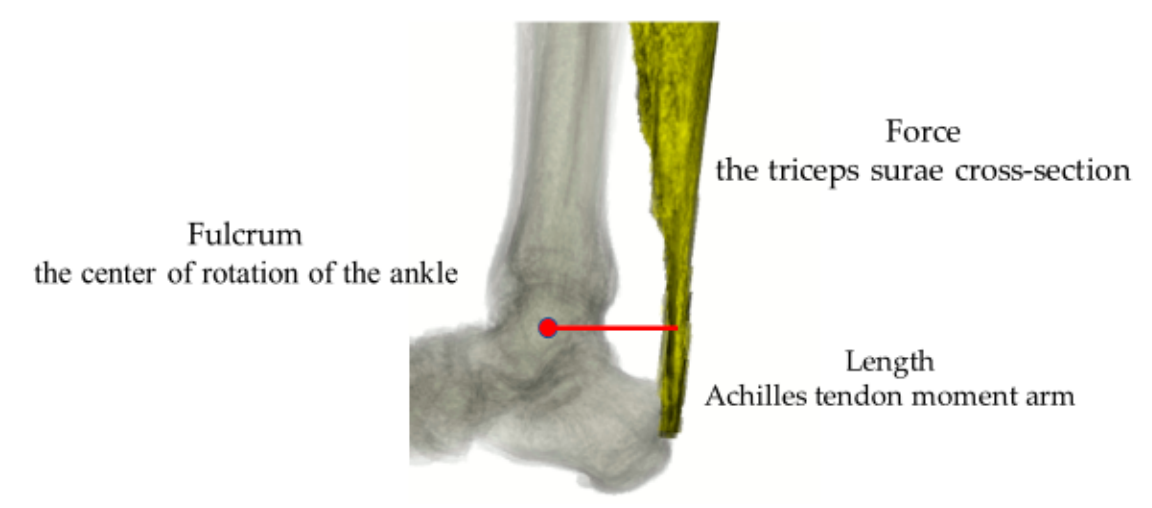

Figure 1. The contraction of the triceps surae can be calculated theoretically as the product of the triceps surae muscle cross-section area and the Achilles tendon moment arm using the lever rule.

The center of rotation (COR) and tendon excursion (TE) methods are mainly used for the measurement of the lever arm of Achilles tendon [5], with MRI and ultrasound generally used for these methods, respectively. Manja et al. [10] proposed a new method using a weight-bearing X-ray. The method allowed the evaluation of the morphology and anatomical position of the bone and soft tissue. Therefore, we are now able to investigate in various patients with Achilles tendon disorders.

Insertional Achilles tendinopathy (IAT), an Achilles tendon disorder, is caused by the traction force of the Achilles tendon [11,12]. This traction force is influenced by the rotational torque of the ankle joint, and the moment arm length is proportional to the plantar flexion torque of the ankle joint [13]. Therefore, when force is applied to the ankle joint, the Achilles tendon moment arm length is inversely proportional to the traction force applied to the insertion of the Achilles tendon. On the other hand, with a short moment arm, the circular arc associated with ankle rotation is thought to shorten, reducing the length displacement of the muscle-tendon complex, and further reducing the consumption of elastic energy of the Achilles tendon $[13,14]$. Thus, the moment arm of the ankle joint is expected to influence the traction force applied to the insertion of the Achilles tendon, the length displacement of the muscle-tendon complex, and the elastic energy.

Surgical treatments for IAT include para-tendon excision, adhesion detachment, intra-tendon degeneration excision, and calcaneal wedge osteotomy [15,16]. Of these, we preferred surgical treatment of insertional Achilles tendinosis with reattachment of the Achilles tendon using the suture bridge technique (Figure 2). This method firmly fixes the enthesis by pressing the tendon with the surface, and good outcomes have been reported with this method [17].

However, due to the lack of basic research on this surgical technique, its effectiveness remains unknown. This study aimed to examine the moment arm length as an anatomical feature in patients with IAT. We hypothesized that the suture bridge method for IAT influences the moment arm length. 

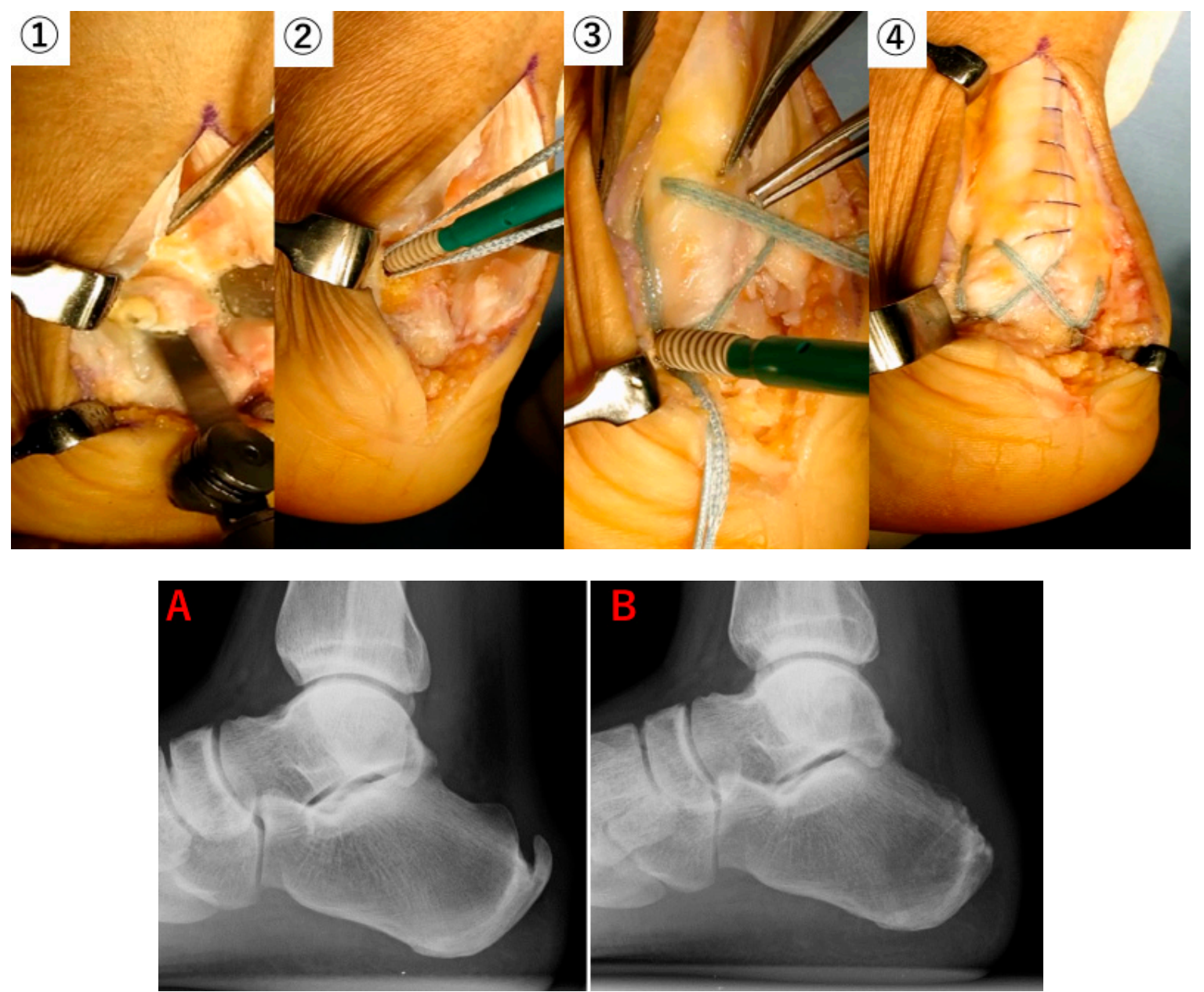

Figure 2. We performed a reconstruction of the Achilles tendon with a suture bridge technique. (1): Bone spurs, calcifications in the tendon, and Haglund's prominence is removed. (2): Two Swive Locks with Fiber Tape inserted in the proximal hole. (3): Fiber Tape is passed through the Achilles tendon and inserted into the prepared distal hole using Swive Lock. (4): The tails on the distal row flushed to the anchor. (A): Preoperative $\mathrm{X}$-ray. (B): Postoperative $\mathrm{X}$-ray for the same case.

\section{Materials and Methods}

This is a retrospective study. Fourteen individuals (16 feet) who required surgical treatment for IAT at our hospital between 2014 and 2019 were included in the IAT group. Those with a history of collagen diseases or trauma other than Achilles tendon injuries, and those who could not be followed up for more than one year after surgery were excluded from the study. As a result, 8 individuals (10 feet; IAT group) were examined in this study. Fifteen healthy individuals (with no complaints of ankle joint injuries nor history of surgical trauma) were included for examination in the control group.

The reconstruction in all IAT cases was performed using the Achilles SpeedBridge system (Arthrex, FL, USA). After extending the Achilles tendon in the midline and partly separating it from the insertion, the osteophytes, intra-tendinous degeneration site, and posterior superior calcaneus at the enthesis were excised. Subsequently, fiber tape was used to fix the Achilles tendon to the calcaneus using four knotless anchors. We analyzed a clinical evaluation by the American Orthopedic Foot and Ankle Society ankle/hindfoot scale (AOFAS scale) and visual analog pain scale (VAS) 1 year after the surgery.

At 6 months to 1 year after the surgery, 1.5T MRI (Vantage Titan Ver3.0, Canon, Tokyo, Japan) images were taken to evaluate the morphology of the Achilles tendon insertion area and abnormal signal in the tendon before and after surgery. 
Plain X-ray images were used to measure the lever arm. The images of the affected side were taken for patients in the IAT group, while those of the healthy side were taken for patients in the control group. As described by Manja et al. [10], who used plain X-ray images of the feet during weight-bearing, the weight-bearing plane was taken as the plantar axis from the lateral image; a straight line perpendicular to this plantar axis, passing through the center of the Achilles tendon, was set as the central axis of the Achilles tendon. The shortest distance from the central axis of the Achilles tendon to the center of the first metatarsal head parallel to the plantar axis was defined as the length of the sole. A perfect circle was drawn to fit the pulley surface of the talus, and the center point was used as the center of motion. The shortest distance from the center of motion to the central axis of the Achilles tendon was defined as the MA length. In addition, the angle between the Achilles tendon moment arm and the line from the center of the ankle joint movement to the insertion of the Achilles tendon was defined as the $\alpha$ angle (Figure 3). The central axis of the Achilles tendon at the time of postoperative measurement was the midpoint from the most dorsal surface to the bottom of the insertion (Figure 4). Also, the ratio of the MA length/foot length was used to standardize the difference in foot size. Furthermore, in order to take the influence of body weight into account, the force applied to the Achilles tendon at the time of heel raise can be calculated as the rotational force around the ankle joint axis. Then, the force applied to the Achilles tendon, F, can be estimated by:

$$
\mathrm{F}=\mathrm{w} \times(\mathrm{L}-\mathrm{MA}) / \mathrm{MA}
$$

where $\mathrm{w}$ is the body weight, MA is the Achilles tendon moment arm length, and $\mathrm{L}$ is the length of the sole $[14,18,19]$ (Figure 5). In the present study, this formula was used to calculate the force applied to the Achilles tendon, and the average value of three measurements performed by one examiner was used.

Both the control group and the IAT group were examined, and all measurements were taken preand post-operatively. Statistical tests were performed using Microsoft Excel. Data were tested using unpaired student $t$-tests, and effects were considered significant at $95 \% \mathrm{CI}$ and $p<0.05$. All subjects gave their informed consent for inclusion before they participated in the study. The study was conducted in accordance with the Declaration of Helsinki, and the protocol was approved by the Ethics Committee of Nara Medical University (Approval number: 2740).

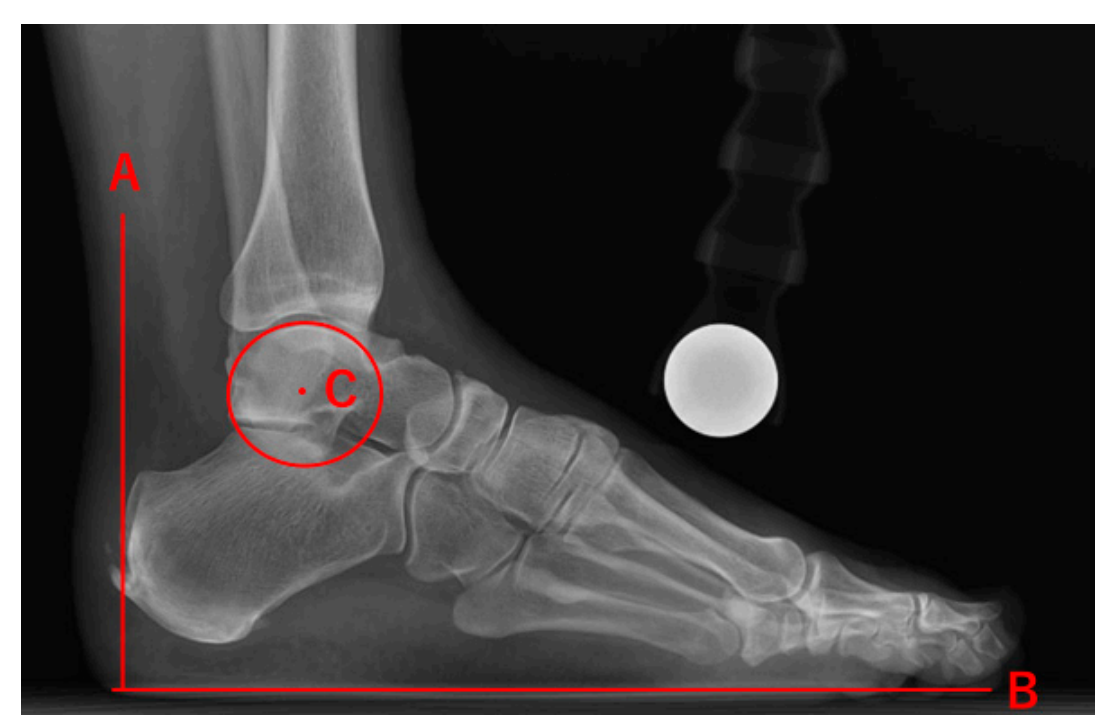

Figure 3. Cont. 


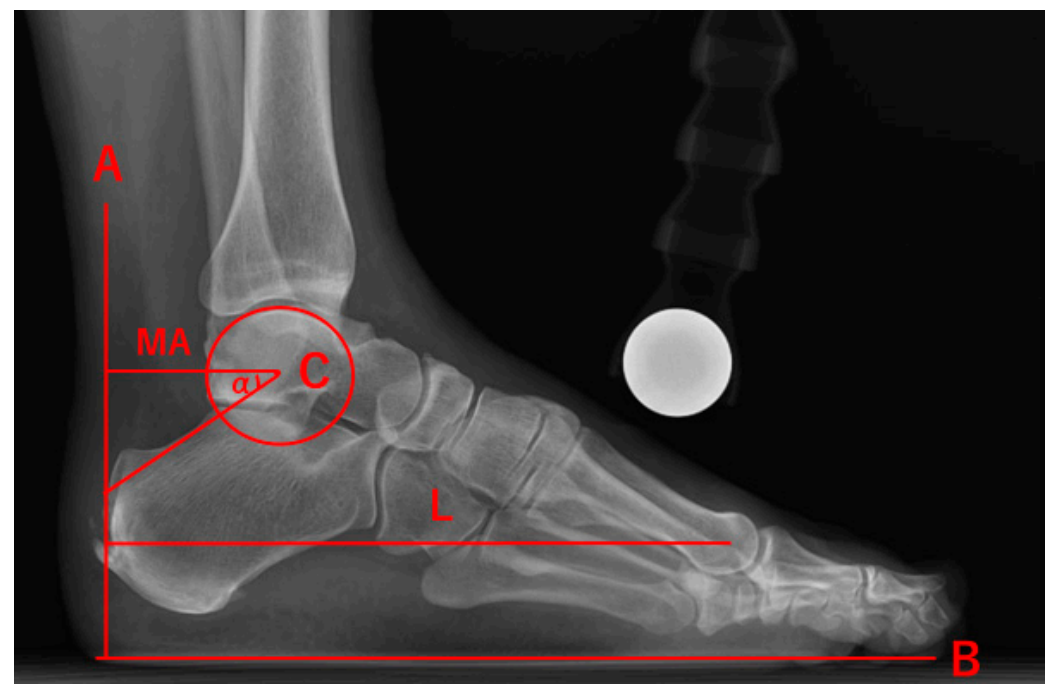

Figure 3. The measurement of the lateral weightbearing radiograph were $(\mathrm{A})$ the centerline of the Achilles tendon insertion, (B) plantar axis, (C) the center of rotation of the ankle, (L) the length of the sole, (MA) the Achilles tendon moment arm $(\alpha)$. The angle between the Achilles tendon moment arm and the line connecting the insertion point and the center of rotation.

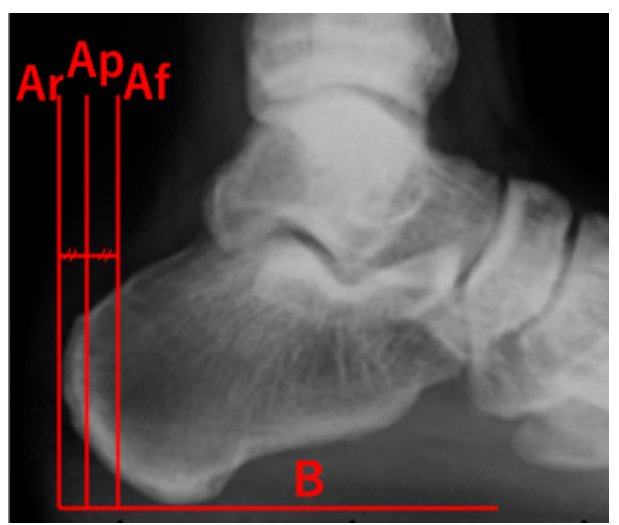

Figure 4. B: Plantar axis. Af: Front edge of the Achilles tendon insertion. Ar: Rear edge of the Achilles tendon insertion. The central axis of Ar and Af was defined as Ap (the central axis of post-operated Achilles tendon insertion).

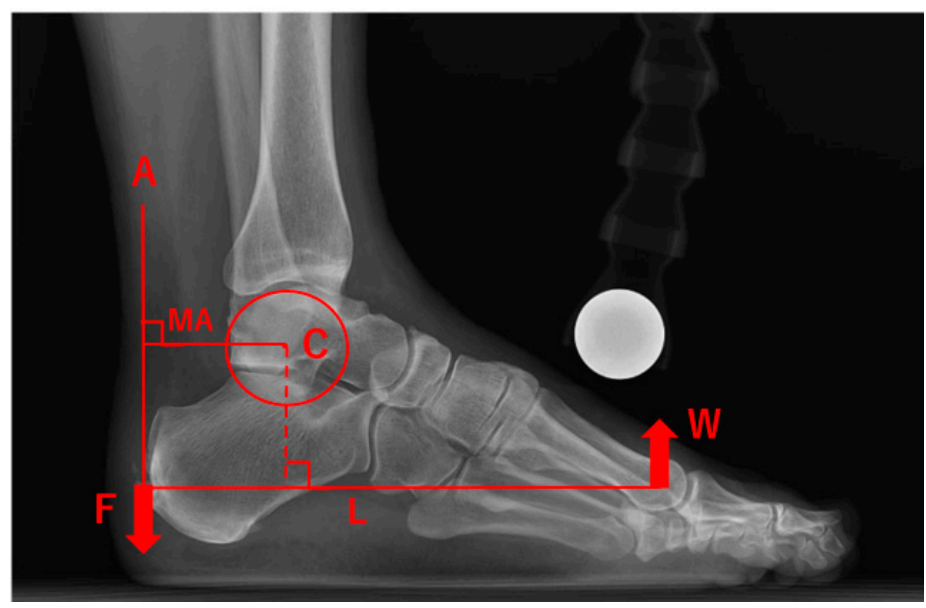

Figure 5. (A) The centerline of the Achilles tendon insertion, (C) the center of rotation of the ankle, (L) the length of the sole, and (MA) the Achilles tendon moment arm. (F) Traction force of the Achilles tendon. (W) Reaction force by body weight. We calculated F using principle of lever. 


\section{Results}

The average height of subjects in the healthy group was $163 \pm 7.4 \mathrm{~cm}$, with average body weight and BMI of $64.5 \pm 12.1 \mathrm{~kg}$ and $24.3 \pm 4.8 \mathrm{~kg} / \mathrm{m}^{2}$, respectively. The average height of subjects in the IAT groups was $163.6 \pm 13.3 \mathrm{~cm}$, with average body weight and BMI of $73.5 \pm 11.0 \mathrm{~kg}$ and $27.7 \pm 4.5 \mathrm{~kg} / \mathrm{m}^{2}$, respectively. Thus, there was no significant difference between the groups for these items (Table 1).

Table 1. Patient Characteristics.

\begin{tabular}{cccc}
\hline & IAT & Control & $p$ Value \\
\hline $\mathrm{n}$ & 10 feet (8 people) & 15 feet (15 people) & \\
Sex & Male: 4 Female: 4 & Male: 8 Female: 7 & \\
Age, $\mathrm{y}$ & $55.5 \pm 14.4$ & $37.4 \pm 16.2$ & \\
Height, $\mathrm{cm}$ & $164 \pm 13.3$ & $163 \pm 7.44$ & $>0.05$ \\
Body weight, $\mathrm{kg}$ & $73.5 \pm 11.0$ & $64.5 \pm 12.1$ & $>0.05$ \\
BMI, $\mathrm{kg} / \mathrm{m}^{2}$ & $27.7 \pm 4.58$ & $24.3 \pm 4.83$ & $>0.05$ \\
\hline
\end{tabular}

Abbreviations: IAT, insertional Achilles tendinopathy. BMI, body mass index. Values are given as mean \pm standard deviation (SD).

The AOFAS scale and VAS improved in all cases (AOFAS scale: Preoperative averaged 71.7, postoperative averaged 92.3; VAS: Preoperative averaged 5.0, postoperative averaged 0.83).

MRI assessment after surgery for IAT revealed Achilles tendon insertion running from the proximal knotless anchor insert to the distal knotless anchor insert via the suture bridge technique in all cases (Figure 6). Also, MRI findings showed the disappearance of abnormal signals in the Achilles tendon in all cases.
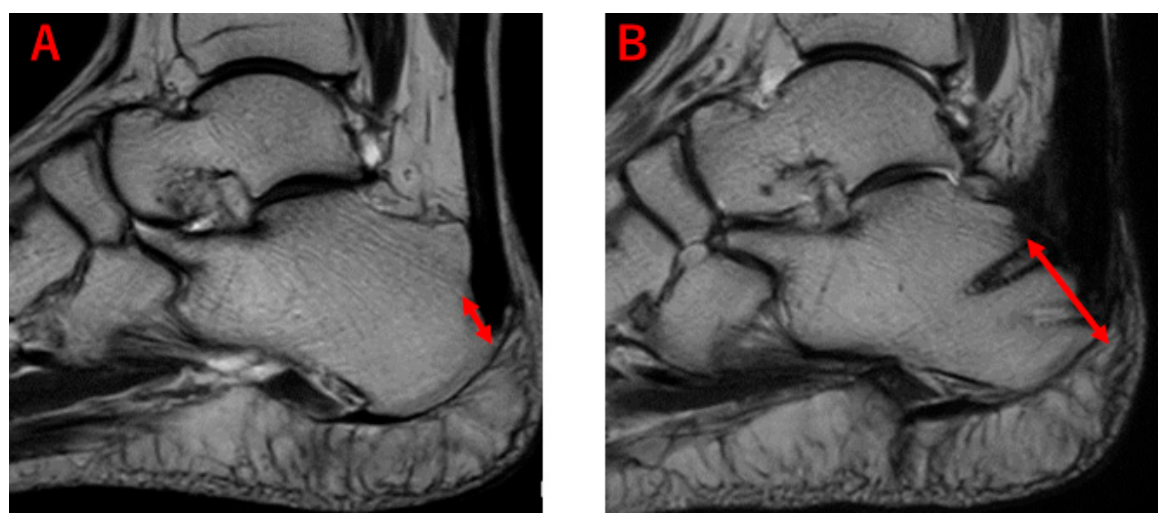

Figure 6. The MRI images of T2-weighted images of the same patient. $\leftrightarrow$ : Length of Achilles tendon insertion. (A): Pre-operation of the Achilles tendon insertion. (B): Post-operation of the Achilles tendon insertion. We can see the postoperative Achilles tendon insertion presence from the proximal knotless anchor insert to the distal knotless anchor insert.

There was no significant difference in the $\alpha$ angle between the control and IAT groups. The ratio of MA length/foot length was $0.30 \pm 0.9 \times 10^{-3}$, in the control group, $0.31 \pm 1.0 \times 10^{-2}$, in the IAT group preoperatively, and $0.27 \pm 0.2 \times 10^{-1}$ in the IAT group postoperatively, showing significant differences between these groups $(p<0.05)$. The force applied to the Achilles tendon was $148.5 \pm 24.9 \mathrm{~kg}$ in the control group, $166.2 \pm 23.0 \mathrm{~kg}$ in the IAT group preoperatively, and $200.8 \pm 25.4 \mathrm{~kg}$ in the IAT group postoperatively. Despite no significant difference in the force between the control and preoperative IAT groups, a significantly higher force to the Achilles tendon was observed in the IAT group postoperatively, compared to the other groups $(p<0.05)$ (Figure 7 ). 

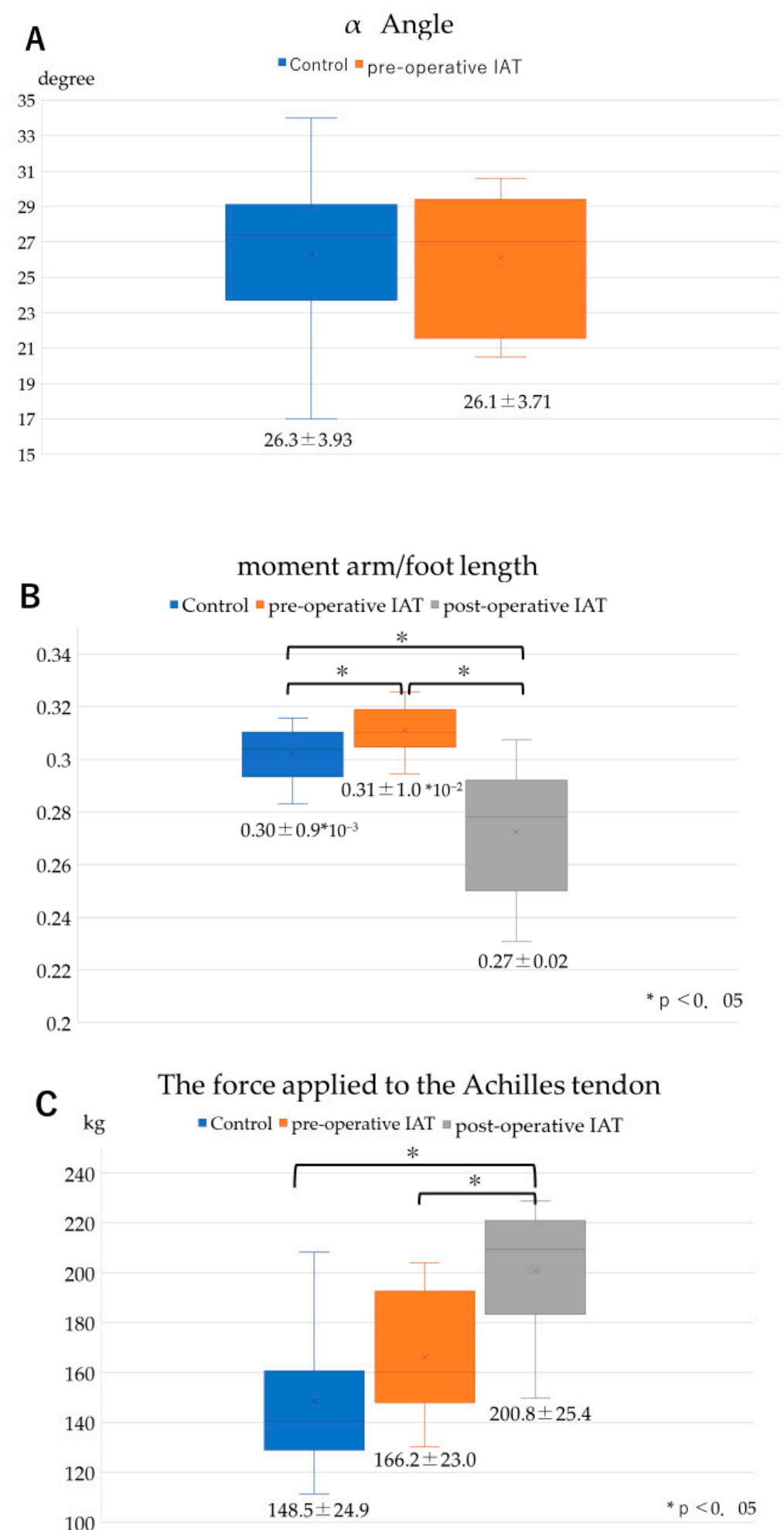

Figure 7. Radiographic Measurement. (A): Comparison of the $\alpha$ angle between the control and pre-operative IAT groups. (B): Comparison of the ratio of MA length/foot length between the control group, the preoperative IAT group, and the postoperative IAT group, showing significant differences between these groups $(p<0.05)$. (C): Comparison of the force applied to the Achilles tendon between the control group, the preoperative IAT group, and the postoperative IAT group, showing that a significantly higher force to the Achilles tendon was observed in the IAT group postoperatively, compared to the other groups $(p<0.05)$. Values are given as mean \pm SD. 


\section{Discussion}

COR method and TE methods are mainly used for the measurement of the lever arm of Achilles tendon [5]. It has been reported that the TE method shows a shorter moment arm than the COR method [5]. However, it has also been reported that there is no difference in measurements between the two methods [20]. Thus, there is no unified view on this issue. Furthermore, the Achilles tendon moment arm has been shown to be influenced by weight-bearing [21], and an evaluation method using weight-bearing X-ray has been developed for taking images during weight-bearing [10]. It is also feasible to use radiographs to measure the Achilles tendon moment arm [10]. In the present study, we examined the group of patients with IAT before and after surgery, in comparison to a control group of healthy individuals. In order to measure the moment arm and evaluation of the morphology of the bone during weight-bearing in each group, we decided to use a weight-bearing $\mathrm{X}$-ray for evaluation. Also, the usage of an X-ray is a simple method and allows us to perform anatomical calculations easily. Therefore, we determined that it was a good method in our study.

A study using this measurement method reported a moment arm length of $64.3 \mathrm{~mm}$ [10]. In the present study, the average moment arm lengths of the healthy and IAT groups were $51.7 \mathrm{~mm}$ and $53.7 \mathrm{~mm}$, respectively, both of which were shorter than the previously reported value. However, the length of the sole of subjects in the previous report was $185.7 \mathrm{~mm}$, in contrast to $171 \mathrm{~mm}$ and $170.1 \mathrm{~mm}$ in healthy and IAT groups, respectively, in the present study. This suggests that the moment arm length may be influenced by the difference in the physical constitution.

In addition, Manja et al. [10] showed that the moment arm length and $\alpha$ angle are influenced by the difference in the shape of the foot, such as flat and concave feet. In the present study, no significant difference in $\alpha$ angle between the control and experimental groups was observed, indicating that the results are not influenced by the difference in the shape of the subject's foot, even in flat and concave feet.

To date, there have been no reports comparing the Achilles tendon moment lengths between healthy individuals and patients with IAT, but many factors are known to influence the pathophysiology of IAT. Lyman et al. [22] stated that it is caused by the traction force of the Achilles tendon during ankle movement. In the present study, those with IAT had a significantly longer Achilles tendon moment arm than healthy individuals. However, there was no significant difference in the force applied to the Achilles tendon between two groups. This indicates that the traction force of the Achilles tendon, which is the cause of IAT, is not influenced simply by the force applied to the Achilles tendon.

It has been shown that the Achilles tendon exerts approximately 4 to 6 times its weight during running [23,24], stretching approximately $8 \%$ of the total length of the Achilles tendon [25]. It has also been shown that, despite the smaller force applied during walking than in running, approximately $7 \%$ of the total length of the Achilles tendon is stretched during walking [26]. This indicates that the insertion of the Achilles tendon is influenced not only by simple tension but also by the elastic energy due to the stretching of the Achilles tendon [27]. In fact, triceps surae eccentric exercise has been recommended as a conservative therapy for IAT [28,29], and the extension of the Achilles tendon is a major cause of IAT. Our data revealed that individuals with IAT had a longer moment arm length than healthy individuals. This suggests that, with a long moment arm, the arc length associated with the ankle rotation lengthens, increasing the length displacement of the muscle-tendon complex, and further increasing the elastic energy consumption of the Achilles tendon [13,14]. Therefore, the elasticity of the Achilles tendon is shown to be greatly responsible for the development of IAT.

In addition, any measurements of the moment arm length before and after surgery using the suture bridge technique have not been reported to date. As a surgical treatment for IAT, the calcaneal wedge osteotomy takes the influence of the moment arm into account and aims to reduce stress on the insertion of the Achilles tendon by shortening the calcaneus length and slightly lifting the insertion of the Achilles tendon [30]. The suture bridge technique used in the present study has also been reported to have good long-term outcomes $[17,31]$. This method can treat various pathological conditions that influence IAT with minimal invasion [31]. Furthermore, by comparing the preoperative and 
postoperative data in this study, the postoperative Achilles tendon moment arm was found to be shortened. On the other hand, the force applied to the Achilles tendon was significantly increased in individuals with IAT compared to healthy individuals. This indicates that this method may not reduce the force applied to the Achilles tendon. Rather, it may reduce the length displacement of the Achilles tendon by reducing the Achilles tendon moment arm, thereby reducing the energy applied to the Achilles tendon.

There are three limitations to this study. First, the number of cases was small. The study needs to proceed with an increased number of cases, and proper statistical analysis needs to be performed. Secondly, the Achilles tendon insertion is an area, but for the sake of simplicity, it was considered to be a point. Performing 3D analyses allows more precise analyses, and we will need to investigate the area of the Achilles tendon insertion. Third, the moment arm measurement method used in this study was the static evaluation. An X-ray or MRI scan is required to measure the Achilles tendon moment arm after the reconstruction of Achilles tendon insertion using the suture bridge technique. However, it is difficult to measure the dynamic change of the moment arm before and after surgery with the measurement methods reported to date. Therefore, a new measurement method needs to be developed for measuring a dynamic moment arm using X-ray or ultrasonic waves in the future.

\section{Conclusions}

In this study, we examined the moment arm in patients with IAT before and after undergoing the suture bridge technique compared to that of healthy individuals. The Achilles tendon moment arm was significantly longer in patients with IAT. We hypothesized that the suture bridge technique for IAT influences the moment arm length. To test this, we examined the changes in the moment arm length of patients with IAT before and after the surgery by analyzing the X-ray images of the lateral side of their feet during under weight-bearing. This study demonstrates that a long moment arm may be one of the causes of IAT, and the suture bridge technique may reduce the Achilles tendon moment arm. These findings suggest that it is important to consider the influence of the Achilles tendon moment arm in the treatment of IAT.

Author Contributions: Conceptualization, Y.S.; methodology, Y.S. and T.M. (Takuma Miyamoto); software, T.M. (Takuma Miyamoto); validation, T.M. (Takuma Miyamoto), Y.S. and Y.T.; formal analysis, T.M. (Takuma Miyamoto); investigation, T.M. (Takuma Miyamoto); resources, Y.T., T.K., A.T., H.K. and T.M. (Tomohiro Matsui); data curation, Y.T., T.K., A.T., H.K. and T.M. (Tomohiro Matsui); writing-original draft preparation, T.M. (Takuma Miyamoto); writing-review and editing, Y.S. and Y.T.; visualization, T.M. (Takuma Miyamoto); supervision, Y.S. and Y.T.; project administration, Y.S. All authors have read and agreed to the published version of the manuscript.

Funding: This research received no external funding.

Conflicts of Interest: The authors declare no conflict of interest.

\section{References}

1. Dayton, P. Anatomic, Vascular, and Mechanical Overview of the Achilles Tendon. Clin. Podiatr. Med. Surg. 2017, 34, 107-113. [CrossRef]

2. Carbone, V.; Fluit, R.; Pelilaan, P.; van der Krogt, M.M.; Janssen, D.; Damsgaard, M.; Vigneron, L.; Feilkas, T.; Koopman, H.F.J.M.; Verdonschot, N. TLEM 2.0-A comprehensive musculoskeletal geometry dataset for subject-specific modeling of the lower extremity. J. Biomech. 2015, 48, 734-774. [CrossRef]

3. Klein Horsman, M.D.; Koopman, H.F.J.M.; van der Helm, F.C.T.; Poliacu Prose, L.; Veeger, H.E.J. Morphological muscle and joint parameters for musculoskeletal modeling of the lower extremity. Clin. Biomech. 2007, 22, 239-247. [CrossRef]

4. Klein, P.; Mattys, S.; Rooze, M. Moment arm length variations of selected muscles acting on talocrural and subtalar joints during movement: An in vitro study. J. Biomech. 1996, 29, 21-30. [CrossRef]

5. Fath, F.; Blazevich, A.J.; Waugh, C.M.; Miller, S.C.; Korff, T. Direct comparison of in vivo Achilles tendon moment arms obtained from ultrasound and MR scans. J. Appl. Physiol. 2010, 109, 1644-1652. [CrossRef] [PubMed] 
6. Maganaris, C.N. Imaging-based estimates of moment arm length in intact human muscle-tendons. Eur. J. Appl. Phsyol. 2004, 91, 130-139. [CrossRef] [PubMed]

7. Olzewski, K.; Dick, T.J.; Wakeling, J.M. Achilles tendon moment arms: The importance of measuring at constant tendon load when using the tendon excursion method. J. Biomech. 2015, 48, 1206-1209. [CrossRef] [PubMed]

8. Csapo, R.; Hodgson, J.; Kinugasa, R.; Edgerton, V.R.; Sinha, S.; Edgerton, V.R.; Sinha, S. Ankle morphology amplifies calcaneus movement relative to triceps surae muscle shortening. J. Appl. Physiol. 2013, 115, 468-473. [CrossRef]

9. Manal, K.; Cowder, J.D.; Buchanan, T.S. Subject-specific measures of Achilles tendon moment arm using ultrasound and video-based motion capture. Physiol. Rep. 2013, 1, e00139. [CrossRef]

10. Manja, D.; Zwicky, L.; Horn, T.; Hintermann, B. The effect of foot type on the Achilles tendon moment arm and biomechanics. Foot 2019, 38, 91-94. [CrossRef]

11. Rufai, A.; Ralphs, J.R.; Benjamin, M. Structure and histopathology of the insertional region of the human Achilles tendon. J. Orthop. Res. 1995, 13, 585-593. [CrossRef] [PubMed]

12. Benjamin, M.; Kumai, T.; Milz, S.; Boszczyk, B.M.; Boszczyk, A.A.; Ralphs, J.R. The skeletal attachment of tendons-tendon 'entheses'. Comp. Biochem. Physiol. A Mol. Integr. Physiol. 2002, 133, 931-945. [CrossRef]

13. Baxter, J.R.; Piazza, S.J. Plantar flexor moment arm and muscle volume predict torque-generating capacity in young men. J. Appl. Physio. 2014, 116, 538-544. [CrossRef]

14. Kunimasa, Y.; Sano, K.; Oda, T.; Nicol, C.; Komi, P.V.; Locatelli, E.; Ito, A.; Ishikawa, M. Specific muscle-tendon architecture in elite Kenyan distance runners. Scand. J. Med. Sci. Sports 2014, 24, e269-e274. [CrossRef]

15. Irwin, T.A. Current concepts review: Insertional Achilles tendinopathy. Foot Ankle Int. 2010, 31, $933-939$. [CrossRef] [PubMed]

16. Chimenti, R.L.; Cychosz, C.C.; Hall, M.M.; Phisitkul, P. Current concepts review Update: Insertional Achilles tendinopathy. Foot Ankle Int. 2017, 38, 1160-1169. [CrossRef]

17. McGravey, W.C.; Palumbo, R.C.; Baxter, D.E.; Leibman, B.D. Insertional Achilles Tendinosis: Surgical Treatment through a Central Tendon Splitting Approach. Foot Ankle Int. 2002, 23, 19-25. [CrossRef]

18. Blazevich, A.J.; Coleman, D.R.; Horne, S.; Cannavan, D. Anatomical predictors of maximum isometric and concentric knee extensor moment. Eur. J. Appl. Physiol. 2009, 105, 869-878. [CrossRef]

19. Carrier, D.R.; Heglund, N.C.; Earls, K.D. Variable Gearing During Locomotion in the Human Musculoskeletal System. Science 1994, 265, 651-653. [CrossRef]

20. Magnaris, C.N.; Balizopolos, V.; Sargeant, A.J. In vivo measurement-based estimations of the human Achilles tendon moment arm. Eur. J. Appl. Physiol. 2000, 83, 363-369. [CrossRef]

21. Rasske, K.; Thelen, D.G.; Prahz, J.R. Variation in the human Achilles tendon moment arm during walking. Comput. Method Biomech. Biomed. Engin. 2017, 20, 201-205. [CrossRef] [PubMed]

22. Lyman, J.; Weinhold, P.S.; Almekinders, L.C. Strain behavior of the distal Achilles tendon: Implications for insertional Achilles tendinopathy. Am. J. Sports Med. 2004, 32, 457-461. [CrossRef] [PubMed]

23. Andrew, G.; Jonathan, S. Comparison of Achilles tendon loading between male and female recreational runners. J. Hum. Kinet. 2014, 44, 155-159. [CrossRef] [PubMed]

24. Willy, R.W.; Halsey, L.; Hayek, A.; Johnson, H.; Willson, J.D. Patellofemoral joint and Achilles tendon loads during overground and treadmill running. J. Orthop. Sports Phys. Ther. 2016, 46, 664-672. [CrossRef]

25. Farris, D.J.; Buckeridge, E.; Trewartha, G.; McGuigan, M.P. The effects of orthotic heel lifts on Achilles tendon force and strain during running. J. Appl. Biomech. 2012, 28, 511-519. [CrossRef]

26. Franz, J.R.; Slane, L.C.; Rasske, K.; Thelen, D.G. Non-uniform in vivo deformations of the human Achilles tendon during walking. Gait Posture 2015, 41, 192-197. [CrossRef]

27. Frankewycz, B.; Penz, A.; Weber, J.; da Silva, N.P.; Freimoser, F.; Bell, R.; Nerlich, M.; Jung, E.M.; Docheva, D.; Pfeifer, C.G. Achilles tendon elastic properties remain decreased in long term after rupture. Knee Surg. Sports Traumatol. Arthrosc. 2018, 26, 2080-2087. [CrossRef]

28. Fahlstrom, M.; Jonsson, P.; Lorentzon, R.; Alfredson, H. Chronic Achilles tendon pain treated with eccentric calf-muscle training. Knee Surg. Sports Traumatol. Arthrosc. 2003, 11, 327-333. [CrossRef]

29. Rompe, J.D.; Furia, J.; Maffuli, N. Eccentric loading versus eccentric loading plus shock-wave treatment for midportion Achilles tendinopathy: A randomized controlled trial. Am. J. Sports Med. 2009, 37, 463-470. [CrossRef] 
30. Perlman, M.D. Enlargement of the entire posterior aspect of the calcaneus: Treatment with the Keck and Kelly calcaneal osteotomy. J. Foot Surg. 1992, 31, 424-433.

31. Greenhagen, R.M.; Shinabarger, A.B.; Pearson, K.T.; Burns, P.R. Intermediate and long-term outcomes of the suture bridge technique for the management of insertional Achilles tendinopathy. Foot Ankle Spec. 2013, 6, 185-190. [CrossRef] [PubMed]

(C) 2020 by the authors. Licensee MDPI, Basel, Switzerland. This article is an open access article distributed under the terms and conditions of the Creative Commons Attribution (CC BY) license (http://creativecommons.org/licenses/by/4.0/). 